\title{
Coherence Properties of Molecular Single Photons for Quantum Networks
}

\author{
Mohammad Rezai, ${ }^{1}$ Jörg Wrachtrup, ${ }^{1,2}$ and Ilja Gerhardt ${ }^{1,2, *}$ \\ ${ }^{1}$ Institute for Quantum Science and Technology $\left(I Q^{S T}\right)$ and $3 r d$ Institute of Physics, \\ D-70569 Stuttgart, Germany \\ ${ }^{2}$ Max Planck Institute for Solid State Research, Heisenbergstraße 1, D-70569 Stuttgart, Germany
}

(Received 22 February 2018; revised manuscript received 10 May 2018; published 26 July 2018)

\begin{abstract}
Quantum mechanics implies that a single photon can be in the superposition of two distant spatial modes and enable nonlocal interferences. The most vivid example is the two-photon coalescence on a 50:50 beam splitter, known as Hong-Ou-Mandel interference. In the past decade, this experiment has been used to characterize the suitability of different single-photon sources for linear optical quantum gates. This characterization alone cannot guarantee the suitability of the photons in a scalable quantum network. As for a deeper insight, we perform a number of nonclassical interference measurements of single photons emitted by a single organic molecule that are optimized by an atomic Faraday filter. Our measurements reveal near unity visibility of the quantum interference, and a one-port correlation measurement proves the ideal Fourier limited nature of our single-photon source. A delayed choice quantum eraser allows us to observe a constructive interference between the photons, and a Hong-Ou-Mandel peak is formed additionally to the commonly observed dip. These experiments comprehensively characterize the involved photons for their use in a future quantum Internet, and they attest to the fully efficient interaction of the molecular photons with a next subsequent quantum node. They can be adapted to other emitters and will allow us to gain insights to their applicability for quantum information processing. We introduce a quality number that describes the photon's properties for their use in a quantum network; this states that effectively $97 \%$ of the utilized molecular photons can be used in a scalable quantum optical system and interact with other quantum nodes. The experiments are based on a hybridization of solid state quantum optics, atomic systems, and all-optical quantum information processing.
\end{abstract}

DOI: 10.1103/PhysRevX.8.031026

Single photons are a crucial ingredient in the field of quantum computation [1,2] and communication [3]. They are the only candidate to practically interlink stationary quantum nodes to an extended network [4] and act as "flying qubits" $[3,5]$. In recent years, a variety of single photons sources were investigated, namely, down-conversion sources [6,7], single atoms or ions [3,8], quantum dots [9-11], and other single emitters [12]. The latter solid state sources hold the promise to be integrated into chip scale devices, to be free of multiphoton contamination. The latter hold the promise to be integrated into chip scale devices, to be free of multiphoton contributions, and to allow for a deterministic single photon generation unlike their parametric downconversion counterparts [11].

An often disregarded, but very versatile single-photon source is single organic dye molecules [12]. They can be

\footnotetext{
*i.gerhardt@fkf.mpg.de
}

Published by the American Physical Society under the terms of the Creative Commons Attribution 4.0 International license. Further distribution of this work must maintain attribution to the author(s) and the published article's title, journal citation, and DOI.
Subject Areas: Optics, Quantum Physics, Quantum Information chemically synthesized such that their emission is aligned to a desired wavelength in the range of 400-800 $\mathrm{nm}$ [12]. At liquid helium temperatures, some candidates are known to emit Fourier limited photons at high flux with tens of $\mathrm{MHz}$ spectral linewidth [13-15] and negligible spectral diffusion. Most importantly, they are likely the single-photon emitters with the highest spectral brightness available, and they do not blink or bleach. A near-unity collection efficiency has been reached under ambient conditions, although the emission is not into a single Gaussian mode [16]. Such a high emission rate should also be achievable for low temperature experiments.

We use the molecule dibenzanthanthrene (DBATT) [Fig. 1(a)], which is dissolved at a low concentration in $n$-tetradecane and subsequently frozen into a Shpol'skii matrix. Its simplified level scheme is depicted in Fig. 1(b). While usual cryogenic single molecule studies excite the molecules resonantly, we focus on the excitation into a vibrational level around $581 \mathrm{~nm}$ and utilize the zero-phonon line photons around $589 \mathrm{~nm}$ for further experiments. This so-called "0-1 excitation" allows us to implement a narrowband single-photon source (down to $12.5 \mathrm{MHz}$ [15]). Furthermore, this off-resonant excitation does not destabilize the emitter nor does it lead to spectral diffusion. 


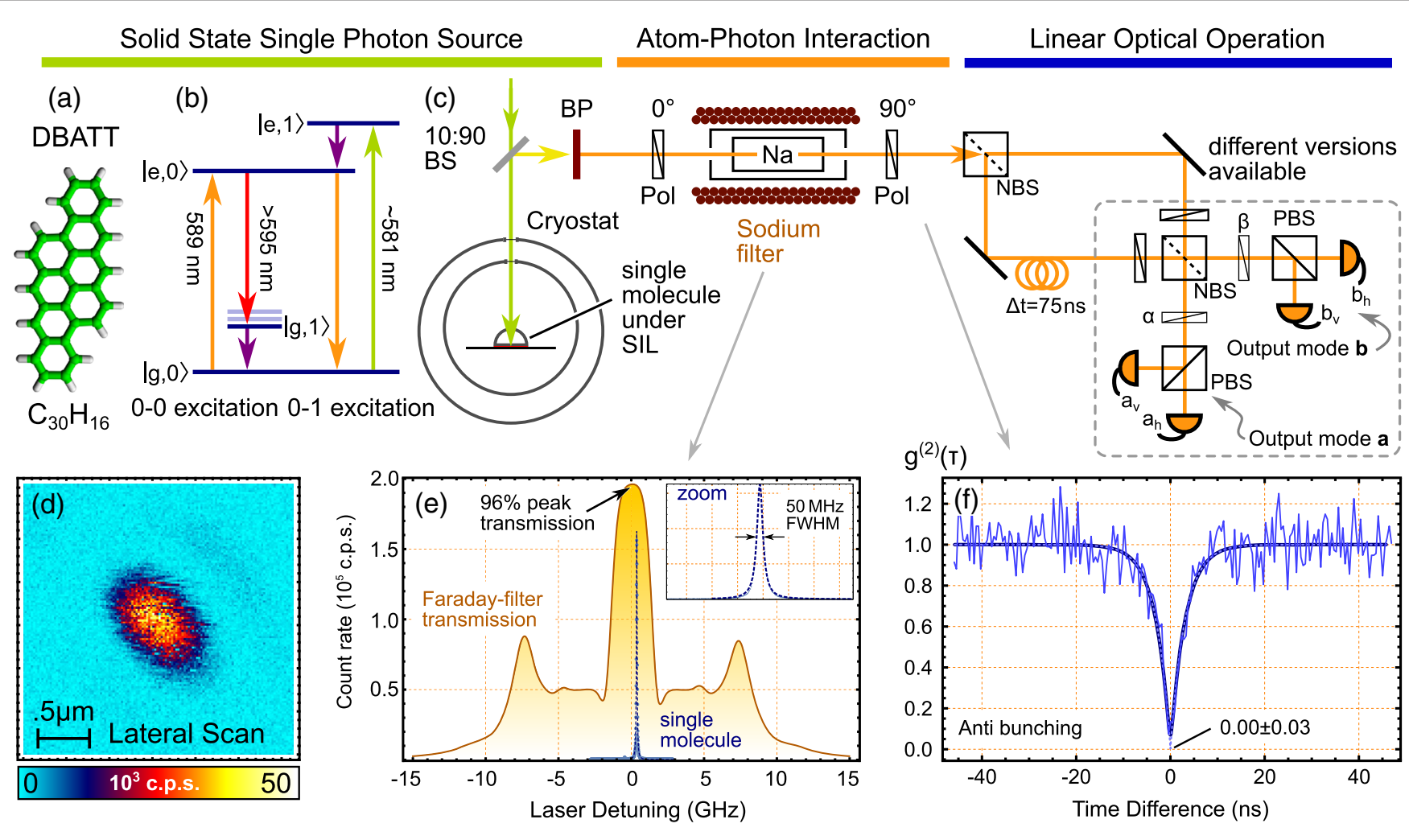

FIG. 1. Experimental configuration. (a) Molecule dibenzanthanthrene (DBATT). (b) Level scheme of the single molecule. (c) Cryogenic confocal microscope, incorporating a solid immersion lens (SIL). The molecular photons are spectral aligned, led through a Faraday filter, and are then fed to an all-optical experiment. In the figure, BP stands for band-pass filter, NBS is the nonpolarizing beam splitter, and PBS is the polarizing beam splitter. (d) Lateral beam scan of the confocal microscope. A single molecule is clearly visible as a bright emitting feature. (e) The atomic vapor Faraday filter opens a few-GHz-wide window with nearunity peak transmission. A molecule matches the atomic sodium spectrum. (f) Antibunching of the purified single molecule emission.

In recent years, we were able to show that the emission is not only bright and narrow-band, but also matches the sodium D-line transitions [14]. This opens the route to quantum hybrid devices that combine the study of true single photons with atomic systems. Being very versatile and robust, hot atomic vapors are ideally suited for this task. They are not only suitable as efficient quantum memories [17], but also allow for the implementation of GHz-wide Faraday filters [15]. The latter are based on the optical interaction of linear polarized light with the nondegenerate Zeeman sublevels in an atomic vapor. The conservation of angular momentum causes the circular components $\sigma_{+}$and $\sigma_{-}$to couple to the increasing and decreasing $m_{F}$ transitions, respectively. As for linear polarized photons, the superposition of $\sigma_{+}$and $\sigma_{-}$leads to a magnetically controlled rotation of the polarization. This is fine-tuned such that only sodium-resonant photons pass through an analyzing polarizer [15]. Figure 1(c) shows the experimental configuration of the filter-it features a 3-GHz passband, as shown in Fig. 1(e). The narrow spectrum of the resonantly excited single molecule is depicted along with the filter spectrum. Its linewidth amounts to $\Delta \nu=50 \mathrm{MHz}$. As neighboring molecules or other background contributions might spoil the emitted single-photon stream [18], the atomic filter is an excellent tool to purify the single-photon emission. Moreover, it selects only the zero-phonon line emission of the manifold of molecular transitions. Since all data from the molecule throughout the paper are presented as raw clicks, the benefits of the filter for quantum information processing can be directly seen in the presented data.

To gain insights to the full temporal behavior of the molecule's emission, the molecule is steadily illuminated. The resulting single-photon stream carries only a single photon at any moment in time. A detector "click" projects a photon to its detection time and thereby eliminates it from the stream. This detection suppresses the probability of a subsequent photon detection-with a time span dominated by the molecule's electronic excited state lifetime ( $\left.\tau_{\mathrm{sp}} \approx 10 \mathrm{~ns}\right)$ [15]. Figure 1(f) shows a recording of the autocorrelation function of the sodium resonant photons that are emitted from the molecule. The fitted value that accounts for the experimental detector jitter amounts to $g^{(2)}(0)=0.00_{-0.00}^{+0.03}$. Subsequently, the single-photon purity is $1.00_{-0.06}^{+0.00}$, defined as $p_{1} / \sum_{n=1}^{\infty} p_{n}$, where $p_{n}$ denotes the normalized probability of receiving $n$ photons. This is outlined in the Supplemental Material [19], Chapter B. The 
source is almost background free, as reported earlier $[14,15]$, and we now extend our view to the interference ability of the photon source.

In a classical view, the above quality measure $\left[g^{(2)}(0) \approx 0\right]$ suggests that the single-photon stream consists of timewise separated particles. This description omits the wave nature of light. Quantum mechanics integrates these two pictures and gives a comprehensive view-the "wave-particle duality." To complement the particle view on our single-photon stream, it is necessary to evaluate its wave nature. This is best characterized by the absolute degree of first order coherence, $\left|g^{(1)}(\tau)\right|$, and is usually an experimental parameter that can be measured in a singlephoton experiment as an interference visibility [20]. In the case of a perfect temporal match of the interfering paths $(\tau=0)$, the value peaks and allows us to estimate the achievable fidelity in a quantum gate. Therefore, in the following, we consider the absolute degree of first order coherence at zero time difference, $\mathcal{C}=\left|g^{(1)}(\tau=0)\right|$, an entity that we would name as "coherentness" of the single photons. This gives a quality number between zero and one to how well the photon's wave nature can be explored. For higher orders, we define the corresponding twophoton property as $\left|g^{(1)}(\tau=0)\right|^{2}$, which characterizes the interference ability in a two-photon experiment, while $\prod_{n=1}^{N}\left|g_{n}^{(1)}(0)\right|$ extends it for $N$ different photons. Some authors have introduced the term "indistinguishability," which is equivalent to $\left|g^{(1)}(0)\right|^{2}$. This is somewhat inconsistent with its use in statistical mechanics where the term "indistinguishable" denotes the intrinsic property of bosons and fermions. While indistinguishability quantifies the outcome of a Hong-Ou-Mandel measurement, $\left|g^{(1)}(0)\right|^{2}$ is the characteristic property of a photon, which can be determined in a variety of ways, as further outlined below (see Supplemental Material [19]).

A well-known experiment in quantum optics that characterizes $\left|g^{(1)}(0)\right|^{2}$ for two photons is the Hong-Ou-Mandel (HOM) interference [6,7]. In this experiment, two photons impinge onto a 50:50 beam splitter and form the two-mode entangled state $(1 / \sqrt{2})\left(\left|2_{a}, 0_{b}\right\rangle+\left|0_{a}, 2_{b}\right\rangle\right)$. A destructive interference renders the probability that the photons are detected at opposite output ports $\left(\left|1_{a}, 1_{b}\right\rangle\right)$ to zero, and therefore, between the two modes, a maximal negative correlation is observed. Such experiments with single emitters, aiming for all-optical quantum computation [1], have been performed in the past decade $[8,20]$ and recently achieved near-unity values of $\left|g^{(1)}(0)\right|^{2}[9,10,21]$. These remarkable results were enabled by their pulsed operation as a "single-photon gun," but this way of recording limits insights into the temporal properties of the photons. When the photons are, e.g., spectral detuned, a quantum beat can be observed, which still shows the destructive interference for zero time difference $[18,22]$.

To study such a two-photon interference in a temporal fine-grained manner, we use the introduced single-photon stream. One option is to use the emission of two independent emitters $[18,23]$. For single molecules with their low spectral diffusion [24], an equivalent and more simple way is the use of a beam splitter, which splits one stream into two identical copies while sacrificing half of the photon flux. The streams are relatively delayed against each other by several coherence lengths to render them independent on the following 50:50 beam splitter. Because of the HOM interference, the photon number at each time point of the output stream is maximally entangled with the corresponding point in the other arm, and $\tau_{c}$, the coherence time of the photons, determines the temporal length of this correlation. As for one arm, we have at each moment the uncertainty between the states $|0\rangle$ and $|2\rangle$. A detection event in one arm (a "click") is indicating the state $|2\rangle$. Such a state collapse witnesses that the other arm is projected to $|0\rangle$ and coincidences are suppressed.

Figure 2, || shows such a correlation measurement between the two output ports. The introduced negative correlation shows up by a suppressed coincidence probability for short measured time differences. At zero time difference, it is reduced down to $g^{(2)}(0)=0.04 \pm 0.03$. In terms of shape, the recording obeys the same temporal behavior as the single-photon antibunching in Fig. 1(f).

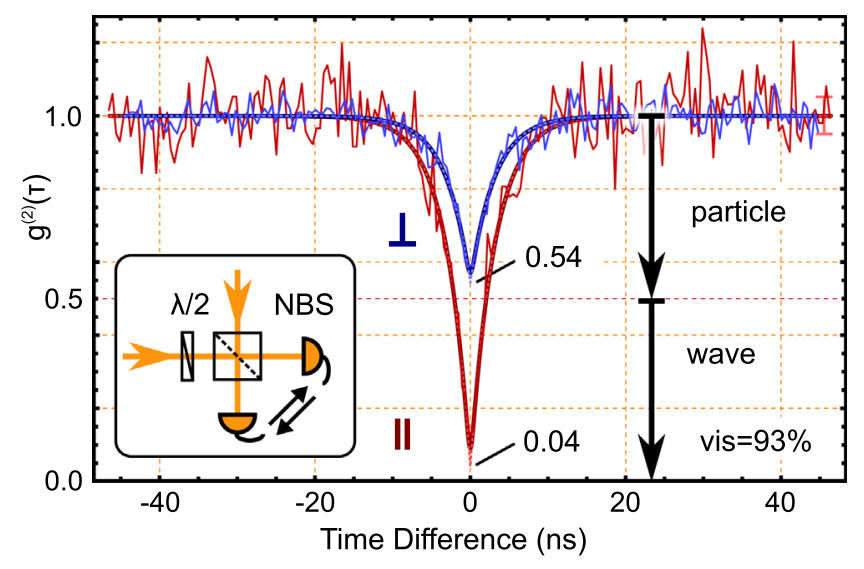

FIG. 2. Two-port correlation $\perp$ : Under an orthogonal polarization input, the two beams cannot interfere and are independent. Nevertheless, with the supplied photons, the time-correlation measurement leads to a correlation down to $g^{(2)}(0)=0.54$ (blue curve). This observed negative correlation is explained by the quantization of the electromagnetic field and matches the description of classical particles. I: Hong-Ou-Mandel configuration; photons in a parallel polarization impinge on the beam splitter (red curve). The above introduced quantization picture is not sufficient to explain the observed result of $g^{(2)}(0)=0.04$. Then, additionally, the superposition of each photon in both output ports becomes relevant. Because of this wave nature, the photons interfere destructively, which reduces the curve from $g^{(2)}(0) \approx 0.5$ down to $g^{(2)}(0) \approx 0$. The visibility of the HOMinterference is calculated as 0.93 [Eq. (2)]. $\lambda / 2$ is the half-wave plate; NBS stands for nonpolarizing beam splitter. 
To gain a more comprehensive view on the Hong-OuMandel interference, a Hanbury Brown and Twiss analysis is added to each of the output ports of the HOM beam splitter. This configuration allows for a measurement of the photon statistics in a single output arm and has been used for a characterization of single photons before [7,25-27]. The corresponding measurement is shown in Fig. 3, $\|$ and shows $g^{(2)}(\tau)=1$ for all measured time differences $\tau$. This becomes clear when we consider that each moment in time carries the uncertainty of detecting zero or two photons, as the one-port state, $\frac{1}{2}(|0\rangle\langle 0|+| 2\rangle\langle 2|)$, indicates. This represents a random intensity fluctuation and correspondingly introduces a positive correlation, where the temporal behavior is bound to the interference ability $\left|g^{(1)}(\tau)\right|^{2}$ with its characteristic coherence time $\tau_{c} / 2$. On the other hand, if we had two noninterfering single-photon streams, a subPoissonian photon statistics with a negative correlation (with a characteristic time $\tau_{\mathrm{sp}} / 2$ ) down to 0.5 would be expected [28]. Both effects-the positive correlation that stems from the interference and the sub-Poissonian dip that originates from the particle picture-completely compensate each other. The effect that any characteristic feature on the one-port HOM measurement vanishes only occurs in the case of perfectly Fourier limited photons $\left(\tau_{c} / \tau_{\mathrm{sp}}=1\right)$. This stems from the fact that only then does the temporal width of the antibunching match exactly the temporal width of the coalescing photons. As outlined in the Supplemental Material [19] (Chapter 8.2), small deviations from the natural linewidth are well witnessed by deviations from the flat line $g^{(2)}(\tau)=1$. In the case of non-Fourier limited photons, the interference is only present for the coherence time of the photons, $\tau_{c}$ [26], which is commonly shorter than $\tau_{\mathrm{sp}}$.

Above, the explanation on the one-port Hong-OuMandel analysis was divided into the particle and wave nature. This equivalently holds for the two-port Hong-OuMandel measurement, where the dip was observed: The particle picture describes a sub-Poissonian statistics down to 0.5 ; however, here, the interference leads to a negative correlation, which "pulls" the value of $g^{(2)}(0)$ down to vanishing values (Fig. 2, $\|$ and arrows).

In conclusion, the wave nature subtracts from or adds to the particle-based curve. For the two-port Hong-OuMandel case, where the curve is pulled downwards, the shape is only the same as the single-photon antibunching, when the Fourier limitation of the photons is guaranteed. However, in the case of the one-port analysis, the Fourier limitation compensates the curve such that the correlation function is $g^{(2)}(\tau)=1$ for all measured time differences. The difference to the particle-type curve is the same for both cases, but with an opposite sign.

As for quantum networking, a spectral match among stationary quantum nodes is essential. If the spectral width of the photons is broader than the natural linewidth of the nodes, the interaction is reduced. This is characterized by the Fourier limitation of the involved photons $\tau_{c} / \tau_{\mathrm{sp}}$ [24]. Subsequently, only well-matching photons efficiently interact with the next quantum node. Hence, so far, such an efficient interaction among remote stationary single emitters has only been demonstrated for atoms [3] and molecules [24].

Besides such a direct interaction in quantum networking, where the Fourier limitation is of importance, another crucial building block is the photonic mediated entanglement among emitters $[3,5]$ and linear optics quantum gates $[1,2]$. The relevant fidelity is estimated by the parameter $\left|g^{(1)}(0)\right|^{2}$. In the two-port measurement (Fig. 2, $\|$ ) it is calculated as $\left|g^{(1)}(0)\right|^{2}=1-2 g_{a b}^{(2)}(0)$, and the experimental value results in $\left|g^{(1)}(0)\right|^{2}=0.92 \pm 0.05$. Here, the depth of the HOM dip is solely limited by dark counts and experimental jitter. Furthermore, for the one-port analysis (Fig. 3, $\|$ ) it is possible to estimate this quality number with the equation $\left|g^{(1)}(0)\right|^{2}=2 g_{a a}^{(2)}(0)-1$, which amounts to $\left|g^{(1)}(0)\right|^{2}=1.00_{-0.08}^{+0.0}$. Both values are consistent with each other.

The above findings show that the photons obey ideal Fourier limitation and interfere efficiently. For quantum information processing, the net overlap of photons with multiple quantum systems (such stationary nodes or other involved photons) is of crucial importance. Hence, we define the quality of the involved photons as $\mathcal{Q}=\left|g^{(1)}(0)\right| \tau_{c} / \tau_{\mathrm{sp}}$, which indicates the achievable match

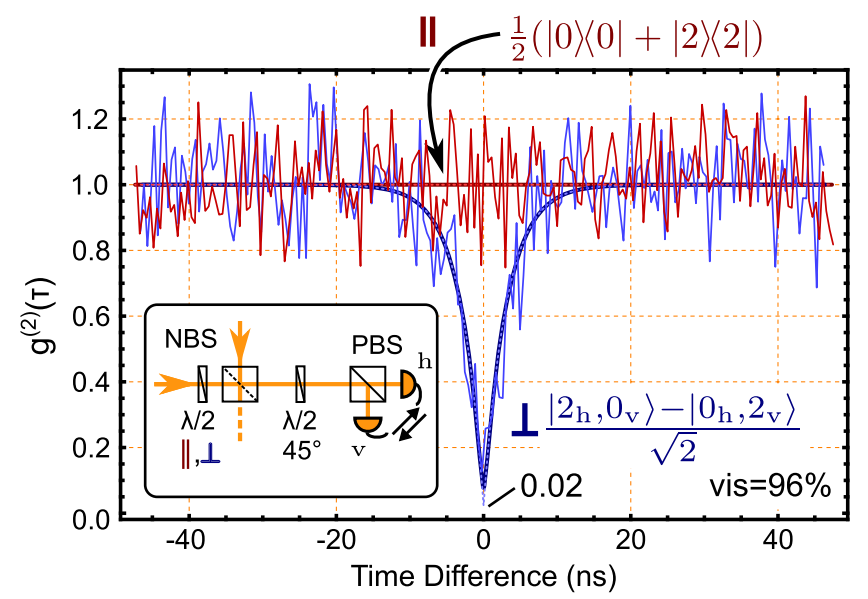

FIG. 3. One-port correlation. \|: The Hong-Ou-Mandel experiment is characterized in a single output arm of the interferometer. The combination of a polarizing beam splitter (PBS) and the halfwave plate $(\lambda / 2)$ acts comparable to a nonpolarizing beam splitter. This recording proves the Fourier limited linewidth by matching $g^{(2)}(\tau)=1 . \perp$ : When the photons are prepared as in the Shih-Alley configuration, the correlation function goes down to zero if the state is analyzed in a diagonal polarization basis. This scheme enables the interference and corresponds to the two-port Hong-Ou-Mandel measurement as in Fig. 2, \|. For the presented data set, $\left|g^{(1)}(0)\right|^{2}$ amounts to 0.96. NBS stands for nonpolarizing beam splitter. 
among photons, and also between photons and stationary quantum nodes. This quality number ranges between zero and one for photons that are emitted from a single quantum emitter. It characterizes the ability of the photons to undergo two- or multiphoton interference, as well as to interact with the next quantum node(s), where the linewidth determines the interaction strength. Both $\left|g^{(1)}(0)\right|^{2}$ and the Fourier limitation, $\tau_{c} / \tau_{\mathrm{sp}}$, affect each other, but each value alone does not guarantee the usability of the photons in a quantum network. Here, $\mathcal{Q}$ amounts to a remarkable $97 \%$, which implies that the photons are suitable for a scalable quantum network with near-optimal photonic-node interaction and efficient all-optical quantum computation.

In comparison of the one- and two-port HOM measurement, one realizes that the correlation within one arm is annulled, whereas between two ports a negative correlation is observed. Pearson's correlation coefficient, defined as $g^{(2)}(\tau)-1$ [29], allows for a mathematical description. For the one-port analysis, it gives a zero value, whereas for the two-port correlation, it results in a maximal negative value of -1 . Since each of the two beams alone does not carry any correlation, it is counterintuitive that, simultaneously, the beams are strongly (negative) correlated. This can be described by a violation of the Cauchy-Schwarz inequality,

$$
\left(g_{a b}^{(2)}(0)-1\right)^{2} \stackrel{\text { class }}{\leq}\left(g_{a a}^{(2)}(0)-1\right)\left(g_{b b}^{(2)}(0)-1\right),
$$

which puts a classical limit to the correlations among two distinct beams, $a$ and $b$. The cross-correlation measurement $g_{a b}^{(2)}(\tau)$ is shown in Fig. 2, $\|$, whereas the one-port correlations $g_{a a}^{(2)}(\tau)$ and $g_{b b}^{(2)}(\tau)$ are equivalent by symmetry and shown in Fig. 3, $\|$. The Cauchy-Schwarz inequality is violated with ratios well above 540. This underlines the nonclassicality of the two-mode entangled state and indicates nonlocal quantum interference. For more information and the relation of the Cauchy-Schwarz inequality to $\left|g^{(1)}(0)\right|^{2}$, we refer to Chapter 10 of the Supplemental Material [19].

In order to investigate the particle picture of the photons, we now suppress their interference. Therefore, the incoming photons are introduced into orthogonal polarization modes, horizontal $(h)$ and vertical $(v)$. This is performed by placing a half-wave plate into one input arm of the HongOu-Mandel beam splitter. This configuration is known as the Shih-Alley configuration [30]. Subsequently, the measurement of the two-port correlation function as well as the one-port correlation results in $g^{(2)}(0)=0.5$.

Figure $2, \perp$ shows the experimental result for this twoport correlation. Now, when the values for $\tau=0$ are compared between the maximally interfering (Hong-OuMandel configuration) and noninterfering photons (ShihAlley configuration), this interference visibility also allows us to determine the joint first order coherence of the two photons by

$$
\left|g^{(1)}(0)\right|^{2}=\frac{g_{\perp}^{(2)}(0)-g_{\|}^{(2)}(0)}{g_{\perp}^{(2)}(0)} .
$$

This results in a remarkable value for a continuous wave experiment of $\left|g^{(1)}(0)\right|^{2}=0.93 \pm 0.05$.

In the Shih-Alley configuration, the two independent input photon streams are set to noninterfering polarizations. When a single output port is considered, they can be again separated by a polarizing beam splitter. All photons from one single-photon stream end up in one output path, whereas all other photons from the second input mode end up in the other one. Therefore, the single-photon streams are totally uncorrelated $\left[g^{(2)}(\tau)=1\right]$. This is different than the one-port Hong-Ou-Mandel measurement, where a similar flat line was observed. Unlike there, such a measurement is fully independent of the wave and particle properties of the input photons.

However, by introducing a wave plate, it is possible to interfere these separable photons. For example, a half-wave plate in front of the polarizing beam splitter in a diagonal basis $\left( \pm 45^{\circ}\right)$ entangles the two polarization modes to the state $(1 / \sqrt{2})\left(\left|2_{h}, 0_{v}\right\rangle-\left|0_{h}, 2_{v}\right\rangle\right)$. This is equivalent to the above Hong-Ou-Mandel transformation, but in polarization modes $(h, v)$ instead of spatial modes $(a, b)$. Consequently, a negative correlation is measured between the output ports of the polarizing beam splitter [8]. This is shown in Fig. $3, \perp$. We like to note that this kind of polarization analysis is limited to the Shih-Alley configuration-when only one polarization mode is occupied (as in HOM), the separation into polarization modes with a polarizing beam splitter is equivalent to a configuration with a nonpolarizing beam splitter (Fig. 3, $\|$ ).

A measurement of such a one-port analysis of the ShihAlley configuration is shown in Fig. $3, \perp$. It shows a reduced probability of coincidences for short measured time differences. As in the two-port Hong-Ou-Mandel scheme discussed above, we estimate $\left|g^{(1)}(0)\right|^{2}=1-2 g_{h v}^{(2)}(0)=$ $0.96 \pm 0.09$. The slight enhancement against the value above is likely a result of the precisely tunable splitting ratio.

As in the two-port study, we compare the results of the one-port measurements in both configurations (Fig. 3, || and $\perp$ ): For the case of parallel incoming photons, no correlation is observed. In the orthogonal input state, the negative correlation is revealed. When the particle picture is considered, a $g^{(2)}(0)=0.5$ is expected. Here, in the one-port correlation, the signal ranges from no correlation $(\|)$ to a maximally negative correlation $(\perp)$. Therefore, the joint first order coherence of the two photons can be determined by

$$
\left|g^{(1)}(0)\right|^{2}=\frac{g_{\|}^{(2)}(0)-g_{\perp}^{(2)}(0)}{g_{\|}^{(2)}(0)+g_{\perp}^{(2)}(0)} .
$$

This results in a value for $\left|g^{(1)}(0)\right|^{2}$ of $0.96 \pm 0.08$. As for the changed denominator against Eq. (2), the relevant 
(a)

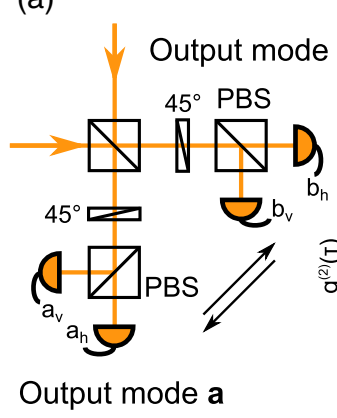

(b) distinghuishable input \& quantum eraser (c) distinghuishable input, delayed choice, non-erased
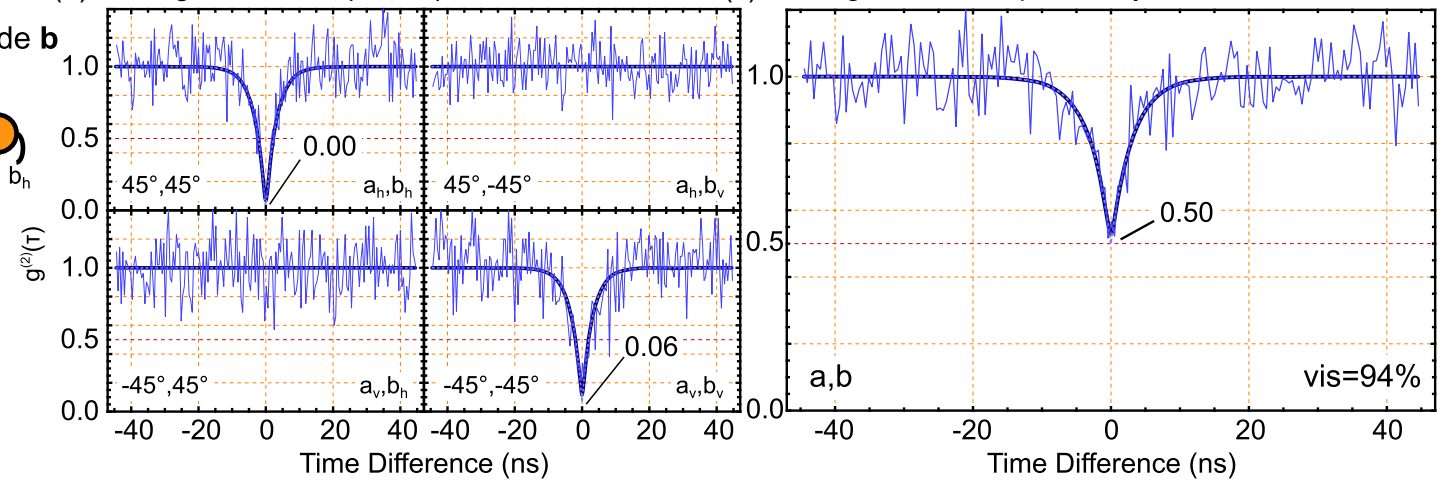

FIG. 4. A delayed choice quantum eraser. In this two-photon interference experiment, the input single-photon streams are prepared as orthogonal, whereas the output modes are analyzed in the $\pm 45^{\circ}$ basis with polarizing beam splitters (PBSs). (a) Experimental setup. (b) Measurement of the cross-correlation signal among the detectors at different output modes $a$ and $b$. Whereas the detection in parallel polarization shows a dip going to zero (like Fig. 2, ||), the orthogonal analysis shows a flat line. (c) When all signals of (b) are added and normalized, the particle-type signal reappears (like Fig. 2, $\perp$ ). For this measurement the two-photon visibility is calculated to 0.94 .

second order correlation functions in this one-port analysis vary between $g^{(2)}(0)=0.0$ and 1.0 , whereas for the twoport analysis, the function changes from $g^{(2)}(0)=0.0$ to 0.5 .

The introduced polarization analysis can be extended to both output ports of the Shih-Alley configuration. To regain the photons' interference, we analyze the photons in the $\pm 45^{\circ}$ basis-i.e., diagonal as they were prepared. The corresponding setup is shown in Fig. 4(a). If we consider the output ports with the parallel polarization $\left( \pm 45^{\circ}\right.$, $\pm 45^{\circ}$ ), the Hong-Ou-Mandel dip is revived as seen in Fig. 4(b). This indicates the destructive interference as introduced above [31] (Fig. 2, \|). On the other hand, when orthogonal polarization is considered $\left( \pm 45^{\circ}, \mp 45^{\circ}\right)$, the corresponding measurements show a flat line. This is a constructive interference, which is associated to a Hong-Ou-Mandel peak [31]. This interference as in the Hong-Ou-Mandel dip occurs between both reflected or both transmitted photons, but due to an additional phase shift, introduced by the polarization difference, the interference is now constructive. This compensates the negative correlation, which is caused by the particle nature of the two photons. Subsequently, the recording shows a flat line in the correlation analysis $\left[g^{(2)}(\tau)=1\right]$. This recording is comparable to the one-port analysis of the Hong-Ou-Mandel interference, which equivalently shows a flat recording and is able to reveal small deviations from the Fourier limitation of the involved photons.

This two-port polarization analysis allows us to revive the Hong-Ou-Mandel interference, although the photons were made incompatible at the beam splitter. The analysis in the $45^{\circ}$ basis erases the which-way information of the detected photons that has been imprinted onto their polarization. This effect has been introduced as a "quantum eraser" [32] and was experimentally implemented for photons from a parametric down-conversion source [31].
It underlines that the photons do not necessarily interfere at the beam splitter, but the heart of the interference is tightly linked to a subsequent detection process [33]. Correspondingly, the wave nature and the interference among two orthogonal polarized photons is evoked by this "postponed compensation" right before detection.

All four curves in Fig. 4(b) are extracted from a single data set that contains time stamps from all four detectors. It is possible to simply disregard the polarization analysis and to consider solely the correlation between the spatial modes $a$ and $b$, as shown in Fig. 4(c). We like to note that this indicates the particlelike nature of the photons and shows a value of $g^{(2)}(0)=0.5$, as recorded in the Shih-Alley configuration (Fig. $2, \perp$ ). Subsequently, we can a posteriori decide whether the path information should be erased from the acquired data or not. This is an implementation of a so-called "delayed-choice quantum eraser" [31,32,34].

This configuration enables a full characterization of the photons in a single measurement, since it integrates all earlier described measurements. $\left|g^{(1)}(0)\right|^{2}$ is best determined when the parallel polarization is analyzed. Here, we measure a value of $\left|g^{(1)}(0)\right|^{2}=0.94 \pm 0.04$, comparable to the Hong-Ou-Mandel case. This includes the single-photon nature, and the photon's usability for all-optical quantum computing [1]. The corresponding recording in the orthogonal polarization is able to reveal small deviations from the Fourier limitation, as discussed for the one-port Hong-Ou-Mandel measurement above. Here, also, a flat line is recorded and confirms the perfect Fourier limitation, which is expressed as $\tau_{c} / \tau_{\mathrm{sp}}=1.0$.

In conclusion, the experiments merge different fields of quantum optics, as the molecular photons from a solid-state source are purified by an atomic vapor and are used in an alloptical operation. Their spectral alignment with atomic sodium is guaranteed by hot atomic vapor via the Faraday effect. Subsequently, all collected photons originate from the 
molecules' zero phonon line and exhibit a unity singleparticle character. This technique has been in parallel successfully adapted to quantum dot single-photon emitters [35].

Beside the above-mentioned single-particle character, we extensively studied the photon's wave nature by a set of two-photon interference experiments. Whereas the usual Hong-Ou-Mandel interference is used routinely to characterize the ability of two photons to interfere, we have applied a one-port correlation experiment that describes the ability of the photons to interact with other quantum systems.

When all measurements above are evaluated together, we determine the joint first order coherence of the two photons as $\left|g^{(1)}(0)\right|^{2}=0.94 \pm 0.03$. This value alone characterizes only the photon's quality in a very specific setting of a quantum gate, but not necessarily in a quantum network. The acquisition under continuous wave laser excitation opens new insights to the temporal coherence.

By this, the photons are proven to be Fourier limited, with a unity value of $\tau_{c} / \tau_{\mathrm{sp}}$. This is experimentally enabled by a recording of a single port of the Hong-Ou-Mandel interference. These measurements reveal the linewidth of the utilized photons to be a few ten MHz. It represents a convenient way to explore the particle and wave nature in a single experiment. Here, since both features are compatible to each other, a flat line, i.e., $g^{(2)}(\tau)=1$, is recorded. This proves the suitability of the photons for a quantum network.

The introduced single-photon source exhibits almost an ideal degree of first order coherence and Fourier limitation. This results in an integrated quality number, $\mathcal{Q}=\left|g^{(1)}(0)\right| \tau_{\mathrm{c}} / \tau_{\mathrm{sp}}$, of larger than $97 \pm 1.5 \%$, and underlines that an efficient optical interaction among flying and stationary qubits in a quantum network is possible-and it guarantees the scalability of quantum gates $[1,2]$ and entanglement distribution [5]. Together with the match to the sodium D-lines, these photons open the route to memory-assisted all-optical quantum gates with an atomic quantum memory. Comparable values of $\mathcal{Q}$ are solely expected for atomic sources [3,8,21], but usually the emission rates are lower due to state preparation steps and a limited photon collection efficiency.

The nature of the Hong-Ou-Mandel interference requires solely that the "which-way" information be absent [33]. As shown, a quantum eraser experiment allows us to revive the interference, even though the photons are incompatible at the location of the beam splitter. The introduced implementation allows for a delayed choice data analysis, since, depending on how this is processed a posteriori, the quantum interference is present or not.

Future experiments will utilize the photons from the single molecule in all-optical quantum computing and entanglement generation $[30,36]$. Furthermore, the photons can be used in implementing a robust and long-lasting storage and retrieval scheme in atomic gases [17,37]—one of the next evident goals in experimental quantum hybridization. The method of utilizing a one-port analysis or a quantum eraser will be used as a further characterization step beyond a characterization in the common HongOu-Mandel experiments.

We thank J. Sperling, W. Schleich, E. Waks, and A. Schell for fruitful discussions. A. Hübner, a stonemason, is acknowledged for the support in preparing the granite table. We further acknowledge the funding from the DFG (Project No. GE 2737/5-1), the MPG, the Sonderforschungsbereich (SFB-Project No. 716), the BMBF, the EU-Project SMeL and the VolkswagenStiftung.

M. R. and I. G. conceived the idea and prepared the experiments. M. R. conducted the experiment and analyzed the experimental results. I. G. and J. W. supervised the team.

The authors declare no competing financial interests.

\section{APPENDIX: METHODS}

\section{Single-photon source}

The single-molecule-based single-photon source is implemented by a cryogenic confocal microscope, as depicted in Fig. 1(c); Fig. 1(d) shows an acquired scan image from the molecule. Light collection is realized by a solid-immersion lens made of cubic zirconia $(\varnothing=3 \mathrm{~mm}$, $n=2.18$ ) in conjunction with an aspheric lens (C330TME, Thorlabs) as in earlier experiments [14]. A sodium resonant single dibenzanthanthrene molecule [DBATT, Fig. 1(a)] is selected from an inhomogeneous distribution of many molecules $(\delta \lambda \approx 1 \mathrm{~nm})$. This is performed by scanning a blue detuned laser $[\lambda \approx 581 \mathrm{~nm}$, Fig. 1(b)] laterally over the sample [Fig. 1(d)], utilizing a sodiumbased Faraday filter [15] in the detection path. Only photons from molecules that are spectral aligned with the sodium transition are transmitted by the filter-no other emitters are visible. The free-space count rate of the emitter is 250 kcounts per second measured after filtering. Then, the light is coupled into a single-mode fiber (Nufern 460HP, antireflection coated input). The coupling efficiency into the fiber amounts to approximately $20 \%$ due to a limited mode overlap; this might be addressed with a mode converter in the form of a spatial light modulator. The final count rate behind the fiber amounts to 50 kcounts per second. A polarization-independent fiber beam splitter guides the photons to two single-photon counters (APDs) for a measurement of the single-photon nature [Fig. 1(f)] or to more complex quantum optical experiments [Fig. 1(c), right].

\section{Atomic vapor filter}

The atomic vapor filter is based on an optical Faraday rotation in atomic sodium vapor with a magnetic field colinearly aligned with the optical axis. A 100-mm-long sodium vapor cell (Triad Technologies, CO, AR-coated inside and outside) resides inside a solenoid. The overall transmission of the cell is $80-85 \%$. The temperature of the 
cell was set to $153^{\circ} \mathrm{C}$ and the utilized magnetic field was around $220 \mathrm{mT}$, which corresponds to approximately $170 \mathrm{~A}$ in the solenoid. For calculating the transmission spectrum, our own program was developed to determine all relevant electric susceptibilities of all-optical transitions [15].

\section{All-optical experiments}

For further nonclassical interference experiments, a lowdrift optical table (granite, weight $=350 \mathrm{~kg}$ ) was dedicated to the experiment. The interferometer is set up with freespace optical elements and single-mode fiber coupled. The entire setup was aligned and characterized by a sodiumresonant and narrow-band ( $\Delta \nu \approx 500 \mathrm{kHz}$ to $1 \mathrm{MHz}$ ) laser prior to the introduced experiments. Since it was performed by visible light (sodium $\mathrm{D}_{2}$ line), tiny interferometric imperfections are directly visible to the eye. When everything is optimally aligned, the interferometric visibility is determined to be larger than $99.9 \%$. The measured optical coupling from the input to the output of the interferometer exceeds $90 \%$ (AR-coated fibers were used). Furthermore, the setup remains stable for days. The incoming photons originating from both arms of the split single-photon stream are delayed longer than their coherence length, so that they are independent $\left(\tau_{\text {delay }} \approx 75 \mathrm{~ns}\right)$.

\section{Data processing}

All data in the paper and the Supplemental Material [19] are presented as raw data. The fit functions were processed with a weighted nonlinear model fit, which has been constrained to physical values (Mathematica 10.4.1, Wolfram Research). For the photon correlation functions, a model including the electrical jitter of the utilized singlephoton detectors was used (solid curves), which depends on the exact cross-correlation jitter of the individual detectors. To derive the theoretical expectation value (dashed curves), the a priori determined jitter was deconvoluted from the fitted model; the data were not corrected for dark counts. For details on the fit with jitter, please refer to the Supplemental Material [19], Chapter A. This paper is accompanied with a Mathematica notebook, which allows us to calculate all relevant (fit) functions.

[1] E. Knill, R. Laflamme, and G. J. Milburn, A Scheme for Efficient Quantum Computation with Linear Optics, Nature (London) 409, 46 (2001).

[2] J. L. O'Brien, G. J. Pryde, A. G. White, T. C. Ralph, and D. Branning, Demonstration of an All-Optical Quantum Controlled-NOT Gate, Nature (London) 426, 264 (2003).

[3] S. Ritter, C. Nölleke, C. Hahn, A. Reiserer, A. Neuzner, M. Uphoff, M. Mücke, E. Figueroa, J. Bochmann, and G. Rempe, An Elementary Quantum Network of Single Atoms in Optical Cavities, Nature (London) 484, 195 (2012).

[4] H. J. Kimble, The Quantum Internet, Nature (London) 453, 1023 (2008).
[5] B. Hensen et al., Loophole-Free Bell Inequality Violation Using Electron Spins Separated by 1.3 Kilometres, Nature (London) 526, 682 (2015).

[6] C. K. Hong, Z. Y. Ou, and L. Mandel, Measurement of Subpicosecond Time Intervals between Two Photons by Interference, Phys. Rev. Lett. 59, 2044 (1987).

[7] J. G. Rarity and P. R. Tapster, Fourth-Order Interference in Parametric Downconversion, J. Opt. Soc. Am. B 6, 1221 (1989).

[8] J. Beugnon, M. P. A. Jones, J. Dingjan, B. Darquié, G. Messin, A. Browaeys, and P. Grangier, Quantum Interference between Two Single Photons Emitted by Independently Trapped Atoms, Nature (London) 440, 779 (2006).

[9] J. C. Loredo et al., Scalable Performance in Solid-State Single-Photon Sources, Optica 3, 433 (2016).

[10] X. Ding et al., On-Demand Single Photons with High Extraction Efficiency and Near-Unity Indistinguishability from a Resonantly Driven Quantum Dot in a Micropillar, Phys. Rev. Lett. 116, 020401 (2016).

[11] H. Wang et al., High-Efficiency Multiphoton Boson Sampling, Nat. Photonics 11, 361 (2017).

[12] B. Lounis and M. Orrit, Single-Photon Sources, Rep. Prog. Phys. 68, 1129 (2005).

[13] A. Kiraz, M. Ehrl, C. Bräuchle, and A. Zumbusch, Ultralong Coherence times in the Purely Electronic Zero-Phonon Line Emission of Single Molecules, Appl. Phys. Lett. 85, 920 (2004).

[14] P. Siyushev, G. Stein, J. Wrachtrup, and I. Gerhardt, Molecular Photons Interfaced with Alkali Atoms, Nature (London) 509, 66 (2014).

[15] W. Kiefer, M. Rezai, J. Wrachtrup, and I. Gerhardt, An Atomic Spectrum Recorded with a Single Molecule Light Source, Appl. Phys. B 122, 38 (2016).

[16] K. G. Lee, X. W. Chen, H. Eghlidi, P. Kukura, R. Lettow, A. Renn, V. Sandoghdar, and S. Götzinger, A Planar Dielectric Antenna for Directional Single-Photon Emission and Near-Unity Collection Efficiency, Nat. Photonics 5, 166 (2011).

[17] O. Katz and O. Firstenberg, Light Storage for One Second at Room Temperature, arXiv:1710.06844.

[18] R. Lettow, Y. L. A. Rezus, A. Renn, G. Zumofen, E. Ikonen, S. Götzinger, and V. Sandoghdar, Quantum Interference of Tunably Indistinguishable Photons from Remote Organic Molecules, Phys. Rev. Lett. 104, 123605 (2010).

[19] See Supplemental Material at http://link.aps.org/ supplemental/10.1103/PhysRevX.8.031026 for the derivation of all relevant equations.

[20] C. Santori, D. Fattal, J. Vuckovic, G. S. Solomon, and Y. Yamamoto, Indistinguishable Photons from a SinglePhoton Device, Nature (London) 419, 594 (2002).

[21] H. P. Specht, J. Bochmann, M. Mücke, B. Weber, E. Figueroa, D. L. Moehring, and G. Rempe, Phase Shaping of SinglePhoton Wave Packets, Nat. Photonics 3, 469 (2009).

[22] T. Legero, T. Wilk, M. Hennrich, G. Rempe, and A. Kuhn, Quantum Beat of Two Single Photons, Phys. Rev. Lett. 93, 070503 (2004).

[23] E. B. Flagg, A. Muller, S. Polyakov, A. Ling, A. Migdall, and G. Solomon, Interference of Single Photons from Two Separate Semiconductor Quantum Dots, Phys. Rev. Lett. 104, 137401 (2010). 
[24] Y. L. A. Rezus, S. G. Walt, R. Lettow, A. Renn, G. Zumofen, S. Götzinger, and V. Sandoghdar, Single-Photon Spectroscopy of a Single Molecule, Phys. Rev. Lett. 108, 093601 (2012).

[25] R. M. Stevenson et al., Quantum Teleportation of LaserGenerated Photons with an Entangled-Light-Emitting Diode, Nat. Commun. 4, 2859 (2013).

[26] J.-H. Kim, C. J. K. Richardson, R. P. Leavitt, and E. Waks, Two-Photon Interference from the Far-Field Emission of Chip-Integrated Cavity-Coupled Emitters, Nano Lett. 16, 7061 (2016).

[27] T. Thomay, S. V. Polyakov, O. Gazzano, E. Goldschmidt, Z. D. Eldredge, T. Huber, V. Loo, and G.S. Solomon, Simultaneous, Full Characterization of a Single-Photon State, Phys. Rev. X 7, 041036 (2017).

[28] R. Loudon, The Quantum Theory of Light, Oxford Science Publications (OUP, Oxford, 2000).

[29] C. C. Gerry, Two-Mode Squeezed Pair Coherent States, J. Mod. Opt. 42, 585 (1995).

[30] Y. H. Shih and C. O. Alley, New Type of Einstein-PodolskyRosen-Bohm Experiment Using Pairs of Light Quanta Produced by Optical Parametric Down Conversion, Phys. Rev. Lett. 61, 2921 (1988).

[31] P. G. Kwiat, A. M. Steinberg, and R. Y. Chiao, Observation of a "Quantum Eraser": A Revival of Coherence in a Two-Photon Interference Experiment, Phys. Rev. A 45, 7729 (1992).

[32] M. O. Scully and K. Drühl, Quantum Eraser: A Proposed Photon Correlation Experiment Concerning Observation and "Delayed Choice" in Quantum Mechanics, Phys. Rev. A 25, 2208 (1982).

[33] T. B. Pittman, D. V. Strekalov, A. Migdall, M. H. Rubin, A. V. Sergienko, and Y. H. Shih, Can Two-Photon Interference Be Considered the Interference of Two Photons?, Phys. Rev. Lett. 77, 1917 (1996).

[34] Y.-H. Kim, R. Yu, S. P. Kulik, Y. Shih, and M. O. Scully, Delayed "Choice", Quantum Eraser, Phys. Rev. Lett. 84, 1 (2000).

[35] S. L. Portalupi, M. Widmann, C. Nawrath, M. Jetter, P. Michler, J. Wrachtrup, and I. Gerhardt, Simultaneous Faraday Filtering of the Mollow Triplet Sidebands with the Cs-D1 Clock Transition, Nat. Commun. 7, 13632 (2016).

[36] D. Fattal, K. Inoue, J. Vučković, C. Santori, G. S. Solomon, and Y. Yamamoto, Entanglement Formation and Violation of Bell's Inequality with a Semiconductor Single Photon Source, Phys. Rev. Lett. 92, 037903 (2004).

[37] K. T. Kaczmarek et al., High-Speed Noise-Free Optical Quantum Memory, Phys. Rev. A 97, 042316 (2018). 